ISSN 2179-6750

\title{
Revisão integrativa: a acupuntura no tratamento da ansiedade e estresse em mulheres com câncer de mama
}

Integration review: acupuncture in the treatment of anxiety and stress in women with breast cancer Revisión integrativa: la acupuntura en el tratamiento de la ansiedad y estrés em mujeres con cáncer de mama

Ana Rita Vieira de Novaes ${ }^{1}$; Camila Brandão-Souza ${ }^{2}$; Eliana Zandonade ${ }^{3}$; Maria Helena Costa Amorim $^{4}$

\section{Resumo}

O câncer de mama é um grave problema de saúde pública, o segundo tipo mais prevalente entre as mulheres e responde por $28,1 \%$ das neoplasias. A busca pelas Práticas Integrativas e Complementares tem aumentado em diversas regiões, e a acupuntura é uma das práticas mais utilizadas da MTC, inclusive na oncologia, com vistas a tratar sintomas do câncer ou dos efeitos adversos do tratamento oncológico. Entre as queixas, encontram-se a ansiedade e o estresse, que causam grandes transtornos. Esta revisão integrativa buscou conhecer, na literatura, informações sobre a aplicação da acupuntura na ansiedade e no estresse de mulheres com câncer de mama. Realizou-se a busca entre julho e novembro de 2015 nas bases de dados da LILACS, MEDLINE, PsycINFO e banco de teses da CAPES, utilizando descritores apropriados. Foram identificados 6 estudos; sendo 3 transversais, 1 descritivo, 1 de coorte transversal e 1 caso controle de base populacional. Os artigos demonstraram a efetividade da acupuntura no tratamento do estresse e da ansiedade no câncer de mama no período per-operatório e durante a quimioterapia. A prevalência do uso da MTC concomitante ao tratamento ocidental é alta, embora a acupuntura seja pouco usada. A desinformação entre os profissionais de saúde ainda perdura e precisa ser mais bem compreendida. Estudar a efetividade em todas as etapas do tratamento oncológico e que avaliem as taxas de recidiva e sobrevida de usuários da acupuntura associada ao tratamento oncológico é indicada.

Descritores: Acupuntura; Ansiedade; Estresse Psicológico; Neoplasias da Mama; Revisão.

\begin{abstract}
Breast cancer is a serious public health problem, the second most prevalent type among women and accounts for $28.1 \%$ of neoplasms. The search for Integrative and Complementary Practices has increased in several regions, and acupuncture is one of the most used practices of TCM, including in oncology, in order to treat cancer symptoms or the adverse effects of cancer treatment. Among the complaints are anxiety and stress, which cause major disorders. This integrative review sought to know, in the literature, information on the application of acupuncture to anxiety and stress in women with breast cancer. The search was carried out between July and November 2015 in the LILACS, MEDLINE, PsycINFO and CAPES databases, using appropriate descriptors. Six studies were identified: 3 cross-sectional, 1 descriptive, 1 cross-sectional cohort and 1 population-based control case. These articles demonstrated the effectiveness of acupuncture in the treatment of stress and

\footnotetext{
${ }^{1}$ Doutoranda em Saúde Coletiva (Área de Concentração: Saúde Coletiva - Epidemiologia) pela Universidade Federal do Espírito Santo (UFES). Coordenação das Práticas Integrativas e Complementares da Secretaria de Estado da Saúde do Espírito Santo. Av. Rio Branco, 1407/1101 - Praia do Canto - Vitória, ES, Brasil. CEP 29055-643. E-mail: novaes.ana2011@gmail.com

${ }^{2}$ Mestre em Ciências da Saúde pela Escola Paulista de Enfermagem (EPE). Enfermeira. Universidade Federal de São Paulo (Unifesp).

${ }^{3}$ Doutora em Estatística pela Universidade de São Paulo (USP). Professora do Programa de Pós-Graduação em Saúde Coletiva. Centro de Ciências da Saúde, Universidade Federal do Espírito Santo (UFES).

${ }^{4}$ Doutora em Enfermagem. Professora aposentada do Programa de Pós-Graduação em Saúde Coletiva. Centro de Ciências da Saúde, Universidade Federal do Espírito Santo (UFES).
} 
ISSN 2179-6750

anxiety in breast cancer in the perioperative period and during chemotherapy. The prevalence of TCM use concomitant with western treatment is high, although acupuncture is poorly used. The disinformation among health professionals still persists and needs to be better understood. To study the effectiveness in all stages of cancer treatment and to evaluate the recurrence and survival rates of acupuncture users associated with oncological treatment is indicated.

Key-words: Acupuncture; Anxiety; Stress, Psychological; Breast Neoplasms; Review.

\section{Resumen}

El cáncer de mama es un grave problema de salud pública, el segundo tipo más prevalente entre las mujeres y responde por el $28,1 \%$ de las neoplasias. La búsqueda por las Prácticas Integrativas y Complementarias ha aumentado en diversas regiones, y la acupuntura es una de las prácticas más utilizadas de la MTC, incluso en la oncología, con miras a tratar síntomas del cáncer o de los efectos adversos del tratamiento oncológico. Entre las quejas, se encuentran la ansiedad y el estrés, que causan grandes trastornos. Esta revisión integrativa buscó conocer, en la literatura, informaciones sobre la aplicación de la acupuntura en la ansiedad y el estrés de mujeres con cáncer de mama. Se realizó la búsqueda entre julio y noviembre de 2015 en las bases de datos de LILACS, MEDLINE, PsycINFO y banco de tesis de la CAPES, utilizando descriptores apropiados. Se identificaron 6 estudios: 3 transversales, 1 descriptivo, 1 de cohorte transversal y 1 caso control de base poblacional. Los artículos demostraron la efectividad de la acupuntura en el tratamiento del estrés y la ansiedad en el cáncer de mama en el período per-operatorio y durante la quimioterapia. La prevalencia del uso de la MTC concomitante con el tratamiento occidental es alta, aunque la acupuntura sea poco usada. La desinformación entre los profesionales de la salud aún perdura y necesita ser mejor comprendida. Estudiar la efectividad en todas las etapas del tratamiento oncológico y que evalúen las tasas de recidiva y sobrevida de usuarios de la acupuntura asociada al tratamiento oncológico es indicada.

Palabras-claves: Acupuntura; Ansiedad; Estrés Psicológico; Neoplasias de la Mama; Revisión.

\section{Introdução}

O câncer de mama é considerado um grave problema de saúde pública no Brasil. É o segundo tipo de câncer mais prevalente entre as mulheres e atualmente responde por $28,1 \%$ do total das neoplasias. A estimativa nacional é de 57.960 casos novos por ano, que representam uma taxa de incidência de 56,2 casos por 100.000 mulheres. É a primeira causa de morte por câncer na população feminina brasileira, com 12,66 óbitos por 100.000 mulheres em 2013. O controle do câncer de mama é hoje uma prioridade da agenda de saúde do Brasil ${ }^{1}$.

A procura pelas Práticas Integrativas e Complementares (PIC), nomenclatura adotada pelo Ministério da Saúde (MS), ou, como denominada pela Organização Mundial de Saúde (OMS), Medicina Tradicional e Complementar (MTC), ou ainda em artigos na literatura internacional Medicinas Alternativas e Complementares (MAC), têm aumentado nas últimas décadas em diversas regiões de todo mundo ${ }^{2,3}$. Segundo a OMS, a acupuntura vem apresentando progressos importantes e atualmente é utilizada em 129 países, reconhecida em $80 \%$ e coberta por seguro-saúde em 18\% dos 
ISSN 2179-6750

casos. É a prática mais utilizada da MTC, cujo aumento observa-se também entre as pessoas com câncer.

Lin et al. ${ }^{4}, 2012$, identificaram a redução dos sintomas de nervosismo e insônia em razão dos efeitos sedativos da acupuntura em pessoas com câncer. Na revisão de Ezzo et al. ${ }^{5}$ evidenciaram-se os benefícios da acupuntura sobre o tratamento medicamentoso sobre as náuseas e vômitos induzidos pela quimioterapia. Em 1998, o Instituto Nacional de Saúde nos EUA afirmou: “A acupuntura é uma modalidade de tratamento com eficácia comprovada para náuseas e vômitos" ${ }^{6}$. Para Spadacio e Barros $^{7}$, essas ferramentas configuram uma opção em potencial para o cuidado em saúde e não podem ser desconsideradas como práticas terapêuticas. Constata-se um crescimento de publicações, entre outros fatores, visando disponibilizar informações aos usuários e à avaliação das intervenções com objetivo de incorporá-las na prática médica tradicional. Para O'Regan e Filshie ${ }^{8}$, a crescente busca pela acupuntura relaciona-se com uma necessidade não satisfeita pela medicina convencional, especialmente pela toxicidade associada com o tratamento oncológico. Segundo os autores, a acupuntura é uma forma eficaz, segura e de baixo custo, que contribui para o autocuidado e reduz o uso de medicações, além de apresentar grande potencial nos cuidados paliativos.

Geralmente os estudos que avaliam o efeito da acupuntura na oncologia são realizados no manejo da sintomatologia da doença, variando com o tipo de tumor e também no controle dos sintomas adversos do tratamento oncológico. A OMS orienta para a importância de integração dos tratamentos tradicionais nos sistemas nacionais de saúde. Recomenda a investigação a fim de proporcionar maior segurança, eficácia e qualidade, assim como o uso racional por profissionais e consumidores ${ }^{2}$.

No Brasil, a acupuntura foi implantada em 1981 no Rio de Janeiro, e posteriormente inserida no Sistema Único de Saúde (SUS) de diversos estados e universidades brasileiras. O reconhecimento como especialidade médica ocorreu em $1995{ }^{9}$. Entretanto, a publicação da Política Nacional de Práticas Integrativas e Complementares (PNPIC) do MS, que orienta as diretrizes para a sua implantação, ocorreu somente em 2006. Essa política recomenda a implementação de ações e serviços no SUS para a prevenção, promoção e recuperação da saúde com métodos não convencionais, além de propor o cuidado continuado, humanizado e integral ${ }^{10}$. Observa-se que entre 2010 e 2012 houve um crescimento de $272 \%$ no número de sessões de acupuntura.

O Programa de Melhoria do Acesso e da Qualidade da Atenção Básica, da Pesquisa Nacional em Saúde (PNS), avaliou 29.798 equipes de Atenção Básica. Dentre as equipes estudadas, 1.583 realizam a acupuntura em 372 municípios ${ }^{11}$. Para Santos e Tesser ${ }^{12}$, este crescimento está relacionado com a valorização da relação médico-usuário; com a tecnologia simplificada e com igual 
ISSN 2179-6750

ou maior eficácia nas situações comuns de adoecimento. Além disso, estimula a autonomia, tendo como categoria central a saúde, e não a doença. Entretanto, observa-se que, apesar deste crescimento e da existência das políticas públicas no campo da acupuntura, ainda é um desafio a implementação de serviços, especialmente em razão do desconhecimento por gestores, reduzido número de profissionais capacitados e insuficientes mecanismos de gestão e financiamento. Além disso, existem os obstáculos, dada a resistência de ordem cultural, assim como as diferenças com o paradigma científico da biomedicina.

Conforme Boaventura Souza Santos ${ }^{13}$, “o paradigma dominante é totalitário, na medida em que nega o caráter racional de todas as formas de conhecimento que não se pautarem pelos seus princípios epistemológicos e pelas suas regras metodológicas". Desta forma, as tensões neste campo continuam presentes e frequentemente as experiências exitosas de serviços de acupuntura no Brasil (e demais práticas integrativas) ainda estão na dependência dos seus representantes sociais.

O estresse psicológico e a ansiedade são frequentes na oncologia. Segundo Cormanique et al. ${ }^{14}$, eventos de vida estressantes são considerados importantes componentes que podem afetar o estado dos indivíduos, e sua associação com a perda de apoio social tem relação com sobrevida significantemente menor em mulheres com câncer de mama. Conforme Mohamed e Baqutayan ${ }^{15}$, pessoas com câncer geralmente enfrentam mais problemas psicológicos em comparação com outros doentes. O diagnóstico do câncer por si já é um grande desafio, além das eventuais incertezas do resultado do tratamento. De acordo com os autores, sintomas de ansiedade, estresse e depressão são mais frequentes no período próximo ao diagnóstico e durante a quimioterapia.

Primo et al. ${ }^{16}$ confirmam que o maior nível de ansiedade ocorre logo após o diagnóstico do câncer de mama, e que a mastectomia é o procedimento mais traumático. Entretanto, sintomas de ansiedade e estresse podem estar presentes durante todas as etapas do tratamento e mesmo após o término, em razão do medo de recidivas e da morte. Esses sintomas podem interferir no sono, causar distúrbios digestivos, respiratórios, cardiovasculares e afetar a qualidade de vida. $\mathrm{O}$ grau de ansiedade varia de uma pessoa para outra, podendo ser mediada por características de personalidade, capacidade de enfrentamento, crença, idade e utilização de estratégias de tratamento da ansiedade. Diante do exposto, buscou-se conhecer na literatura informações sobre a aplicação da acupuntura na ansiedade e no estresse de mulheres com câncer de mama.

\section{Métodos}

Trata-se de uma revisão integrativa sobre as implicações da acupuntura no estresse e na ansiedade em mulheres com câncer de mama.<smiles>[14CH2][14CH2][Mg]</smiles> 


\section{Estratégia de busca}

Para a elaboração da revisão, inicialmente houve a identificação do tema e a seleção da hipótese e, posteriormente, a fixação de critérios de inclusão e de exclusão de estudos, definiu-se quais informações deveriam ser extraídas e a categorização dos estudos, avaliaram-se os estudos incluídos, interpretaram-se os resultados e, por fim, apresentou-se a síntese dos achados ${ }^{17}$. As estratégias de busca estão representadas na Tabela 1.

Tabela 1. Estratégia de busca dos artigos, dissertações e teses originais

Estratégia de busca dos artigos originais

Breast Neoplasms AND Acupuncture OR

Acupuncture Therapy

Acupuncture OR Acupuncture Therapy AND Anxiety OR Anxiety Disorders

Acupuncture OR Acupuncture Therapy AND stress

Acupuncture AND Preoperative AND Breast Neoplasms

Estratégia de busca das dissertações e teses

Acupuntura e Câncer de mama

10.105

298

10

LILACS PsycINFO

MEDLINE

LILACS

P

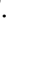

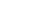


ISSN 2179-6750

Realizou-se a busca dos estudos pela Biblioteca Virtual da Saúde (BVS) e pelo motor de busca PubMed, bem como no banco de teses da Coordenação de Aperfeiçoamento de Pessoal de Nível Superior (CAPES), entre os meses de julho e novembro de 2015. Utilizaram-se as bases de dados da Literatura Latino-Americana e do Caribe em Ciências da Saúde (LILACS), da Medical Literature Analysis and Retrieval System Online (MEDLINE), e da American Psychological Association (PsycINFO), mais importante base de dados da psicologia, sendo os operadores booleanos utilizados “AND” e "OR", e os termos: "Breast Neoplasms AND Acupuncture OR Acupuncture Therapy", "Acupuncture OR Acupuncture Therapy AND Anxiety OR Anxiety Disorders", “Acupuncture OR Acupuncture Therapy AND stress" e "Acupuncture AND Preoperative AND Breast Neoplasms", na respectiva ordem. Para teses e dissertações do banco da CAPES os termos foram: "Acupuntura e Câncer de mama", "Acupuntura e Ansiedade", "Acupuntura e Estresse" e "Acupuntura e Préoperatório".

Estratégia de análise e seleção

Foram incluídos artigos originais, teses e dissertações disponibilizados na íntegra e na forma online, publicados nos idiomas português, inglês ou espanhol, sem restrição quanto ao período de publicação, necessariamente com mulheres diagnosticadas com câncer de mama inclusas na amostra e que em algum momento do tratamento fizeram uso da acupuntura. Foram excluídas revisões bibliográficas, artigos de atualização, comunicações, formulação de protocolos, reflexão e artigos repetidos nas diferentes bases de dados (Figura 1).

Inicialmente considerou-se o idioma de publicação do estudo. Logo após, levou-se em conta o título do trabalho e sua proximidade ao tema escolhido e posteriormente a adequabilidade dos resumos à proposta desta pesquisa. Em seguida, avaliou-se de maneira integral cada estudo selecionado. Nessa fase houve análise profunda de cada artigo por duas revisoras, uma médica acupunturista e uma enfermeira com formação em câncer de mama, ambas mestres. Os dados importantes e mais relevantes de cada estudo são apresentados de forma resumida na Tabela 2. 
Figura 1. Fluxograma dos artigos selecionados para análise.

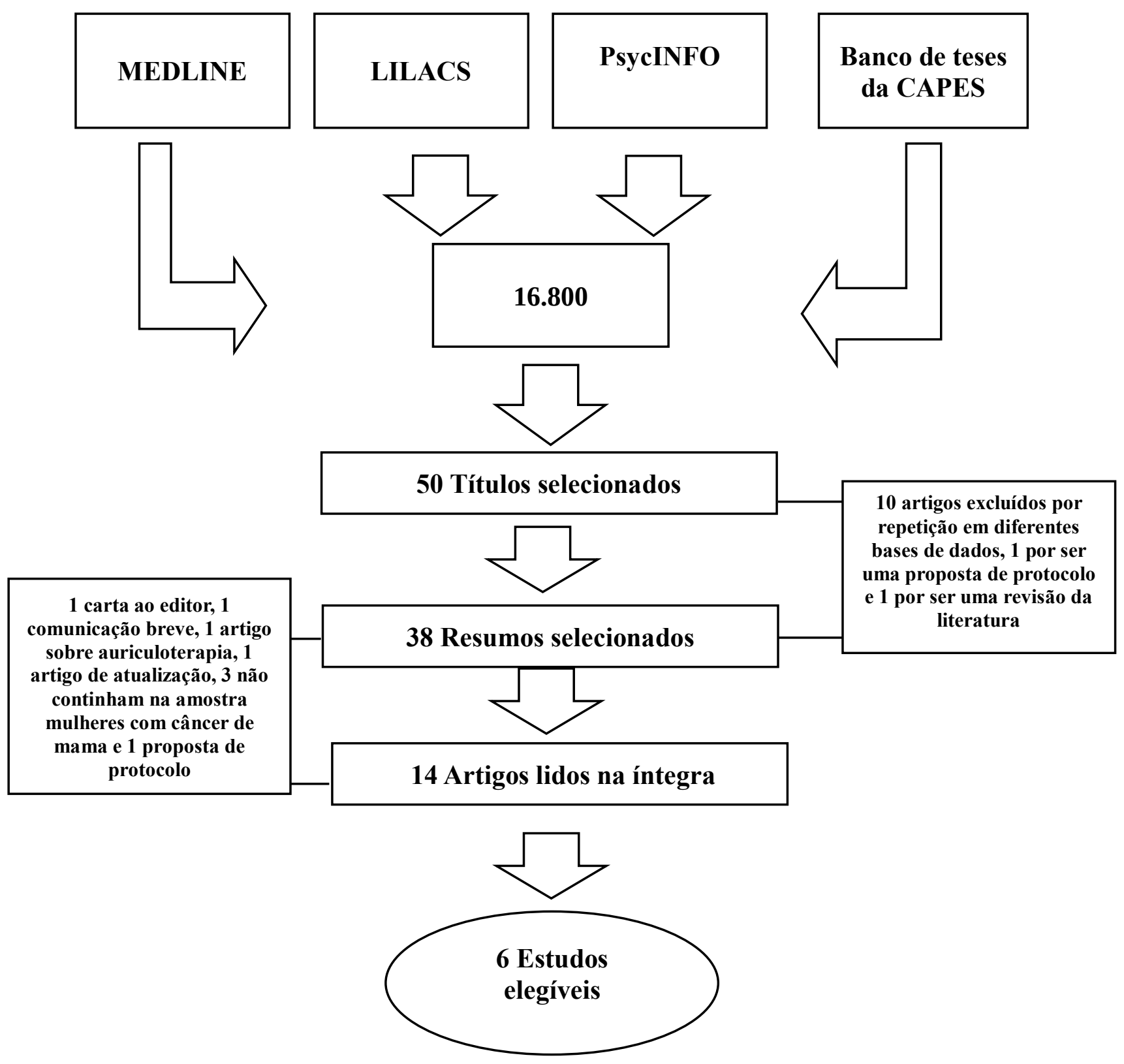

Fonte: As autoras (2017). 
Tabela 2. Categorização dos estudos

\begin{tabular}{|c|c|}
\hline Número & 1 \\
\hline Autores & Molly J. Mallory e col. \\
\hline Título/Periódico & $\begin{array}{c}\text { Acupuncture in the Postoperative Setting for Breast } \\
\text { Cancer Patients: A Feasibility Study In: The } \\
\text { American Journal of Chinese Medicine }\end{array}$ \\
\hline Ano/Tipo/Local & 2015 - Estudo transversal - EUA \\
\hline Objetivos & $\begin{array}{c}\text { Avaliar a viabilidade da acupuntura em hospital e o } \\
\text { efeito sobre o estresse, ansiedade e dor no pós- } \\
\text { operatório. }\end{array}$ \\
\hline População & $\begin{array}{l}20 \text { mulheres com câncer de mama submetidas à } \\
\text { mastectomia ou reconstrução. }\end{array}$ \\
\hline Metodologia & $\begin{array}{l}\text { Avaliou-se a intensidade dos sintomas antes e após a } \\
\text { acupuntura manual individualizada. Utilizou-se o } \\
\text { REDCap e cálculos pelo teste t pareado. }\end{array}$ \\
\hline Resultados e conclusões & $\begin{array}{l}\text { Idade média de } 49,50 \text { anos, IMC } 25,4 \mathrm{~kg} / \mathrm{m} 2 ; 90 \% \\
\text { são caucasianos, } 80 \% \text { casados; } 50 \% \text { nunca fumaram, } \\
\text { 95\% fariam novamente acupuntura e } 100 \% \text { a } \\
\text { recomendariam. } 70 \% \text { avaliou a experiência como } \\
\text { melhor que o esperado. Ansiedade, } \\
\text { tensão/desconforto e dor apresentaram melhora } \\
\text { significante após a acupuntura. }\end{array}$ \\
\hline
\end{tabular}

\section{Número}

\section{Autores}

Título/Periódico

\section{Ano/Tipo/Local}

Objetivos

População

Metodologia

Resultados e conclusões

\section{2}

Mackereth Peter, Bardy Joy, Filshie Jacqueline, Finnegan-John Jennifer, Molassiotis Alexander

Receiving or not receiving acupuncture in a trial: The experience of participants recovering from breast cancer treatment. In: Complementary Therapies in Clinical Practice

$$
2014 \text { - Estudo descritivo - Inglaterra }
$$

Explorar a experiência de doentes com câncer de mama em uso da acupuntura.

$$
40 \text { participantes }
$$

Estudo qualitativo realizado com grupo focal e análise de discurso.

As participantes expressaram desespero com o esgotamento das possibilidades de tratamento para fadiga e avaliaram a acupuntura como algo que lhes trazia prazer, relaxamento, calma, energia, melhora

do sono e do humor, bem-estar, diminuição de linfedema e dores. No grupo controle a reação foi de tristeza e frustração. 


\begin{tabular}{lc}
\multicolumn{1}{c}{ Número } & 3 \\
Autores & Yong Cui, Xiao-Ou Shu, Yutang Gao, Wanqing \\
& Wen, Zhi-Xian Ruan, Fan Jin, Wei Zheng \\
Título/Periódico & Use of complementary and alternative medicine by \\
Chinese women with breast cancer In: Breast \\
Cancer Research and Treatment.
\end{tabular}

Ano/Tipo/Local

Objetivos

População

Metodologia

Resultados e conclusões

2004 - Estudo caso-controle de base populacional China

Avaliar a prevalência e consumo da MTC, motivações e percepção de eficácia e relações demográficas e clínicas entre mulheres com câncer de mama.

1.065 mulheres de 25 a 64 anos participantes do Shanghai Breast Cancer Study.

Entrevistas a 1.065 mulheres quanto ao uso de acupuntura, fitoterapia, suplementos, exercícios e grupos de apoio. Variáveis: idade, estado civil, renda, escolaridade, estádio, tempo do diagnóstico, metástase/recidiva e protocolo de tratamento. Análises pelo Statistical Analysis Software e teste qui-quadrado ou exato de Fisher's.

Idade média de 48 anos, $93 \%$ casadas, $86,2 \%$, estádio 0- II seguimento de 4,3 anos. Recidiva em $8,5 \% .98 \%$ utilizavam MTC, $86,4 \%$ a fitoterapia, $4,9 \%$ a acupuntura, $84,8 \%$ os suplementos, $65,5 \%$ exercícios, $16,6 \%$ grupos de apoio, $51,2 \%$ três ou mais terapias. Motivação principal foi o tratamento do câncer. $48,1 \%$ relataram eficácia da acupuntura, $7,7 \%$ pela modulação do sistema imunológico, com diminuição da dor e sintomas da menopausa. A maioria associa a MTC com tratamentos ocidentais.

Número
Autores
$\begin{gathered}\text { Thi Chen e col. } \\ \text { Título/Periódico Use of Complementary and Alternative } \\ \text { Medicine Among Chinese Women with Breast } \\ \text { Cancer. In: The Journal Of Alternative and } \\ \text { Complementary Medicine. }\end{gathered}$

Ano/Tipo/Local

Objetivos

População

149
2008 - Estudo de coorte transversal - China

Prevalência e percepção dos benefícios da MTC e a relação com fatores demográficos e clínicos.

5.046 participantes do Shanghai Breast Cancer Study 


\section{Metodologia}

Resultados e conclusões

\section{Ano/Tipo/Local}

Objetivos

População

Metodologia

Resultados e conclusões
Aplicou-se questionário estruturado em mulheres com câncer de mama que usaram a acupuntura, fitoterapia, suplementação, exercício, grupos de apoio por no mínimo $1 \mathrm{x} / \mathrm{sem} / \mathrm{mês}$.

Idade média de 53,5 anos, $51 \%$ pós-menopausa, $71,4 \%$ com sintomas da menopausa, $54 \%$ com alta escolaridade, $88 \%$ casadas, $85,6 \%$ estadiamento 0 -II,

$73 \%$ estavam satisfeitas com a qualidade de vida, 97,2\% utilizaram a MTC, 77,2\% a suplementação, $76,8 \%$ a fitoterapia e $0,4 \%$ a acupuntura. $\mathrm{O}$ uso entre as chinesas com câncer de mama é alto e concorre com tratamentos convencionais.

\begin{tabular}{lc}
\multicolumn{1}{c}{ Número } & 5 \\
Autores & D Tas, D Uncu, M Sendur, N Koca and N Zengin \\
Título/Periódico & Acupuncture as a Complementary Treatment for \\
& Cancer Patients Receiving Chemotherapy In: Asian \\
Pacific Journal of Cancer Prevention Prev.
\end{tabular}

Investigar a eficácia da acupuntura na ansiedade, dor, náuseas, vômitos e qualidade do sono em pessoas hospitalizadas sob quimioterapia.

18 mulheres registradas no Oncology Department of Numune Hospital

Variáveis: diagnóstico, sexo, escolaridade. Excluídos: febre alta, infecção, sangramento, trombocitopenia e uso de medicação anticoagulante. As sessões foram realizadas um dia antes, no dia e no dia após a quimioterapia. Avaliação da ansiedade foi feita pelo Beck Anxiety Scale, da dor por Escala

Analógica Visual, intensidade das náuseas e vômitos, questionário graduado e para insônia o Insomnia Severity Index. A avaliação foi feita no primeiro e quarto dia de internação e análise estatística pelo SPSS.

Houve redução de $72,2 \%$ da náusea, $52,8 \%$ do vômito, $15,6 \%$ da ansiedade e da prevalência para $2,2 \%$, totalizando uma diminuição de $57,8 \%$ no total. A insônia teve redução geral, e $11 \%$ da insônia grave foi eliminada. A dor teve diminuição em 53,3\%. Houve melhora da dor, ansiedade, insônia, náuseas e vômitos. Observou-se melhoria com apenas uma única sessão de acupuntura. 


\begin{tabular}{lc}
\multicolumn{1}{c}{ Número } & 6 \\
Autores & Pu CY, Lan VM, Lan CF, Lang HC \\
Título/Periódico & $\begin{array}{c}\text { The determinants of traditional Chinese medicine } \\
\text { and acupuncture utilization for cancer patients with } \\
\text { simultaneous conventional treatment In: European } \\
\text { Journal of Cancer Care }\end{array}$ \\
Ano/Tipo/Local & 2008 - Estudo - transversal Taiwan \\
Objetivos & $\begin{array}{c}\text { Investigar os determinantes do uso da MTC e da } \\
\text { acupuntura em pacientes com câncer tratados } \\
\text { simultaneamente pela medicina convencional. }\end{array}$ \\
População & 366 mulheres com câncer de mama \\
Metodologia & Aplicados questionários com variáveis \\
& socioeconômicas e demográficas com regressóes \\
logísticas para as categorias, as variáveis \\
dependentes são MTC e acupuntura.
\end{tabular}

A maioria possuía união estável, escolaridade alta e baixa renda. Para o uso da acupuntura, a religião não

foi determinante. Pessoas com ensino superior tiveram 4,8 vezes mais probabilidade de usarem a acupuntura, assim como o sexo feminino. As

Resultados e conclusões mulheres com câncer são 55,5\% mais propensas a associarem o procedimento com seus tratamentos convencionais. Embora a MTC seja secular, ainda é desconhecida por muitos médicos em Taiwan.

Fonte: As autoras (2017).

\section{Resultados}

Nesta revisão foram selecionados seis estudos publicados entre 2004 e 2015, dos quais três em revistas de medicina complementar e três em revistas de oncologia. Em relação aos locais de realização da pesquisa, quatro foram desenvolvidos no Oriente, e os demais um no Reino Unido e outro nos EUA. Quanto ao tipo de estudo, três foram transversais, um descritivo, um coorte transversal e um caso-controle de base populacional. Para a realização desta revisão integrativa, incluíram-se todos os artigos que se referiam direta ou indiretamente à temática, visando responder a pergunta proposta de forma mais abrangente.

Os objetivos de três dos estudos ${ }^{18-20}$ foi avaliar os padrões de consumo das medicinas não convencionais, as motivações e as percepções de eficácia e a relação entre a clínica e o perfil demográfico. Yong et al. ${ }^{18}$ e Zhi Chen et al. ${ }^{19}$ utilizaram os dados do Shanghai Breast Cancer Study. 
ISSN 2179-6750

Yong et al. ${ }^{18}$ entrevistaram 1.065 participantes entre 25 a 64 anos que utilizaram de forma contínua pelo menos um tipo de terapia pelo período de no mínimo um mês após o diagnóstico de câncer de mama. Entre as usuárias da MTC a idade média foi de 48,1 anos, (93\%) casadas, $(86,2 \%)$ entre os estadiamentos 0 - II. Todas as mulheres se submeteram a cirurgia e a quimioterapia e $(39,3 \%)$ a radioterapia. Entre elas (98\%) utilizavam algum tipo de MTC: a fitoterapia (86,4\%), os suplementos em $(84,8 \%),(65,5 \%)$ praticavam exercícios, $(16,6 \%)$ frequentavam grupos de apoio e $(4,9 \%)$ a acupuntura. Neste grupo $(51,2 \%)$ utilizavam três ou mais terapias.

As motivações para a procura pela acupuntura foi considerar o tratamento eficaz (46\%) e (7,7\%) para auxiliar na modulação do sistema imunológico, na redução dos sintomas da dor e demais desconfortos e atenuação das queixas relacionadas a menopausa. A maioria das chinesas com câncer de mama utiliza a MTC associada a outros tratamentos ocidentais e relataram benefícios terapêuticos. Zhi Chen et al. ${ }^{19}$ aplicaram um questionário estruturado a 5.046 mulheres entre 20 e 75 anos. Considerou-se como usuária da MTC/acupuntura e fitoterapia, suplementação alimentar, atividade física e grupos de apoio, mulheres que utilizaram, após o diagnóstico de câncer de mama, um tipo de terapia, no mínimo uma vez por semana durante um mês. Os resultados mostraram que a idade média foi de 53,5 anos, (51\%) pós-menopausadas e $(71,4 \%)$ com sintomas da menopausa. Dessas $(53,6 \%)$ apresentaram elevado grau de escolaridade. As casadas totalizaram (87,9\%), e $(99,8 \%)$ das mulheres fizeram cirurgia. Destas, (49,3\%) apresentaram receptores hormonais de estrogênio e progesterona positivos, e $(85,6 \%)$ encontravam-se entre o estadiamento 0 e II. Quase todas as participantes (97,2\%) relataram uso de pelo menos uma das modalidades da medicina tradicional, sendo a suplementação a mais comumente usada $(77,2 \%)$, seguida pela fitoterapia $(76,8 \%)$, e somente $(0,4 \%)$ das mulheres usaram a acupuntura. Segundo os autores, o uso da medicina alternativa por chinesas com câncer de mama é alto e concorre com tratamentos convencionais.

$\mathrm{Pu}$ et al. ${ }^{20}$ investigaram os determinantes do uso da MTC e da acupuntura em mulheres com câncer de mama. Evidenciou-se que (22\%) dos pacientes utilizavam a MTC e (5\%) utilizaram acupuntura associada ao tratamento convencional. Houve predomínio de mulheres, casadas, e as religiões prevalentes foram o budismo e o taoísmo. A maioria possuía renda mensal baixa. A maior escolaridade foi registrada entre as mulheres com câncer de mama. Observou-se maior probabilidade do uso da MTC e da acupuntura nos casos de câncer de mama. Pacientes com maior tempo de evolução do câncer tiveram maiores probabilidades de utilizarem a MTC. Para o uso da acupuntura, a religião não foi determinante. Pessoas com ensino superior tiveram 4,8 vezes mais probabilidade de usarem a acupuntura. A renda e a idade (mais jovem) foram determinantes importante no uso da MTC. O mesmo não foi verdadeiro para a acupuntura. $\mathrm{O}$ sexo feminino foi determinante importante para o uso da acupuntura. As mulheres com câncer são $(55,5 \%)$ mais propensas 
ISSN 2179-6750

a associarem o procedimento com seus tratamentos convencionais. Apenas 57 pacientes utilizaram a MTC e a acupuntura simultaneamente ao tratamento convencional.

O estudo de Mallory et al. ${ }^{21}$ teve como objetivos avaliar a viabilidade da acupuntura em ambiente hospitalar e investigar o tratamento com acupuntura sobre o estresse, ansiedade e dor no pós-operatório de mulheres que foram submetidas a mastectomia ou a cirurgias de reconstrução mamária em razão do câncer de mama. Foram realizadas sessões diárias desde o primeiro dia do pósoperatório até a alta. Para mensuração dos efeitos e para avaliar a satisfação com o tratamento foi aplicado no início e no final da intervenção o Was-it-Worth-it Questionnaire (WIWI) e a Symptom Visual Analogic Scale (VAS). O tratamento baseou-se na teoria da MTC de "Acalmar o shen, tonificar e mover o Qi e o sangue".

Os pontos utilizados foram Baihui (GV20) para acalmar a mente, Yintang (EX-HN3) para eliminar a dor de cabeça e acalmar a mente, Hegu (LI4) para eliminar a dor de cabeça e promover analgesia, Quchi (LI11) para promover analgesia no ombro, extremidade superior e abdome, Neiguan (PC6) para acalmar a mente, harmonizar o estômago e aliviar a náusea e o vômito, Xuehai (SP10) para mover o sangue e aliviar a estase sanguínea, Sanyinjiao (SP6) para tonificar o yin e o sangue e harmonizar o fluxo de qi no fígado, Zusanli (ST36) para eliminar a dor no trato gastrointestinal e aliviar a náusea e vômitos, Taichong (LV3) para clarear o fluxo de qi e reduzir a dor e tensão no tórax e Qiuxu (GB40) para reduzir a dor, tensão no tórax e hipocôndrio e eliminar a dor de cabeça. Foram usadas agulhas de aço inoxidável com inserção profunda até promoverem a sensação de "de qi" (sensação de dor, dormência ou distensão). Os dados foram inseridos no REDCap.

As participantes possuíam a média da idade de 49,5 anos, Índice de Massa Corpórea de 25,4 $\mathrm{kg} / \mathrm{m}^{2} ;(90 \%)$ eram caucasianos, $(80 \%)$ casados. Neste grupo (90\%) inferiram a acupuntura como um tratamento positivo e $(95 \%)$ participariam novamente da intervenção. Todos os usuários recomendariam a acupuntura, (70\%) avaliou a experiência como melhor que o esperado. Todos os sintomas avaliados, a ansiedade $(p=0: 0065)$, a tensão e o desconforto muscular $(p=0: 0001)$, e a dor $(\mathrm{p}=0: 0023)$, apresentaram melhora significante considerando o pré e o pós-tratamento com a acupuntura.

Demet Tas et al. ${ }^{22}$ investigaram a eficácia da acupuntura na ansiedade, dor, qualidade do sono e sintomas digestivos em mulheres com câncer de mama internadas e submetidas à quimioterapia. As sessões de acupuntura foram realizadas por três dias consecutivos, antes, durante e depois da quimioterapia. Para mensurar a dor utilizou-se a Escala Analógica Visual, enquanto que para avaliar a intensidade das náuseas e vômitos, utilizou-se questionário graduado entre 0 e 4 . Para a insônia utilizou o questionário Insomnia Severity Index e para a ansiedade, o Beck Anxiety Scale. Neste 
ISSN 2179-6750

estudo encontra-se referência dos pontos de acupuntura utilizados, PC6, H7, ST36 e LIV3, bilateralmente, uso de agulhas metálicas e 20 minutos de duração das sessões. Os resultados demostraram uma diminuição significante da náusea $(72,2 \%)$, vômito $(52,8 \%)$, da ansiedade $(57,8 \%)$, insônia (queda geral, sendo que 11\% da insônia grave foi eliminada) e da dor, que apresentou uma redução de (53,3\%), comparando o pré e o pós-tratamento.

Peter et al. ${ }^{23}$ exploraram por meio dos grupos focais as experiências de mulheres que usaram a acupuntura pós-quimioterapia. As mulheres verbalizaram o desespero para enfrentar a fadiga e referiram prazer, relaxamento e calma com a acupuntura. As participantes do grupo intervenção consideraram excelente o tratamento, tendo sido capaz de trazer boas memórias, mais energia, melhora do sono, humor, bem-estar, disposição para o exercício, diminuição de linfedema e alívio de dores. No grupo controle a reação foi de decepção, tristeza e frustração por não receberem a acupuntura.

\section{Discussão}

Observa-se que nesta revisão não houve uniformidade entre os tipos de estudos desenvolvidos nem na metodologia utilizada, não sendo possível qualquer análise estatística.

Mallory et al. ${ }^{21}$ apresentaram um ensaio clínico para avaliar o efeito da acupuntura no pósoperatório de mulheres com câncer de mama. Apesar da pequena amostra $(n=20)$, os resultados do estudo de Mallory et al. ${ }^{21}$ sugerem que o emprego da acupuntura em ambiente hospitalar é viável e os resultados apresentados demonstraram significante melhora da ansiedade, dor, tensão e maior relaxamento no pós-operatório. Embora mencionem que a seleção dos pontos tenha sido feita de forma individualizada, segundo as bases da MTC, os mesmos não esclarecem de forma precisa de que forma foram utilizados, como por exemplo se parcialmente ou em sua totalidade, ou se houve necessidade de acréscimo de algum outro ponto, uma vez que se considerou a necessidade do indivíduo.

Observou-se também um elevado grau de satisfação dos participantes com o tratamento, como demonstrado pelo percentual de pessoas que relataram que participariam novamente de uma intervenção (95\%). Este dado demonstra a importância do acesso a essa prática no pós-operatório. A apresentação dos resultados não segue as recomendações da STRICTA, documento que visa contribuir para a sistematização e melhoria da qualidade dos relatórios dos ensaios clínicos de acupuntura ${ }^{24}$. Ressalta-se a importância de estudos que avaliem intervenções usadas no préoperatório com vistas a melhorar as condições clínicas dos doentes, uma vez que o estresse préoperatório e a ansiedade afetam até $80 \%$ das pessoas. Além disso, produz consequências negativas 
ISSN 2179-6750

no intra e no pós-operatório, dificultando o controle da dor e os níveis de náuseas e vômitos. Em outro estudo realizado por Volkan Acar et al. ${ }^{25}$, utilizando um único acuponto, Yintang, pode-se observar redução significante dos níveis de ansiedade (STAI $p<0,018$ ). Shu-Ming Wang et al. ${ }^{26}$ também obtiveram resultados satisfatórios utilizando a auriculoterapia para tratar a ansiedade no préoperatório $(\mathrm{p}<0,014)$.

Demet Tas et al. ${ }^{22}$ utilizaram a acupuntura durante a quimioterapia, empregando vários instrumentos para avaliar os sintomas adversos do tratamento quimioterápico como ansiedade, insônia, dor, náuseas e vômitos. Neste estudo há menção aos pontos de acupuntura utilizados, o tempo de duração das sessões e o tipo de agulhas utilizadas. Os achados demonstram que apenas uma única sessão de acupuntura foi suficiente para beneficiar os pacientes, com melhora para todos os desfechos. Destaca-se a importância do tratamento com acupuntura, considerando a sensibilidade para promover a redução importante da ansiedade (57,8\%), com eliminação dos quadros graves de insônia. Além disso, mais da metade das usuárias relataram redução das algias e de sintomas digestivos, (72,2\%) da náusea $(52,8 \%)$ e vômito.

Confirma-se a hipótese de que a acupuntura pode ser útil nos centros oncológicos, possui baixo custo e que há menos efeitos secundários do que os tratamentos convencionais. Outros estudos 25-27 demonstraram que a melhoria da ansiedade e do estresse puderam ser obtidos com técnicas e diferentes grupos de pontos. Segundo a MTC, para a prescrição do tratamento para a acupuntura, deve-se considerar o padrão de desarmonia do indivíduo. Assim sendo, existem vários pontos que podem ser utilizados para o tratamento de um mesmo sintoma, ou de grupos de sintomas e nosologias. Desta forma justifica-se que diferentes estudos adotem planos terapêuticos diversos.

Estudos de prevalência do uso da MTC, como os de Zhi Chen et al. ${ }^{19}$ e Yong et al. ${ }^{18}$, incluem outras modalidades de técnicas, além do consagrado tratamento da MTC. Ou seja, na relação de abordagens da MTC, inseriu-se o uso de suplementos, exercícios físicos e grupos de apoio. Ressaltase que não há detalhamento que explicite exatamente o que são e a forma de utilização desses recursos. Segundo a revisão sistemática de Ernst e Cassileth et al. ${ }^{27}$, a prevalência do uso de CAM no câncer em adultos variou de 7 a 64\%. Destacou-se a grande variabilidade de terapias e a necessidade de melhor compreender conceitos como de "medicina complementar / alternativa" por parte de investigadores e usuários. Conforme Spadacio e Barros ${ }^{7}$, "esse conjunto de práticas alternativas e complementares necessita ser diferenciado entre as racionalidades e técnicas terapêuticas, pois significa a incorporação de elementos de outra racionalidade médica. A homeopatia, a medicina ayurvédica (assim como a Medicina Tradicional Chinesa), por exemplo, possuem outra doutrina médica explicativa do que é a doença ou o adoecimento, origem ou causa, evolução ou cura." 
ISSN 2179-6750

E conclui, "as demais práticas são apenas técnicas e portanto muito facilmente incorporadas como complementares aos tratamentos convencionais”. Assim, a inclusão de práticas que possuem grandes diferenças, tais como as encontradas nesses estudos, pode dificultar a comparação e análise e influenciar nos resultados apontados.

Um obstáculo para a compreensão do nível de renda da população oriental presente nos estudos é o uso da moeda chinesa como padrão de referência. Ressalta-se que não há menção quanto à presença de efeitos adversos ou possíveis interações entre a fitoterapia chinesa e os quimioterápicos. Entretanto, as interações entre esses tratamentos constituem preocupação de autores, sugerindo uma avaliação sistemática da eficácia e segurança das ervas medicinais a fim de identificar possíveis modificações de efeitos. De fato, conforme o Conselho Federal de Farmácia $(\mathrm{CFF}){ }^{28}$, há vários estudos que demonstram que fitoterápicos podem potencializar ou reduzir o efeito de inúmeras drogas. Como, por exemplo, substâncias metabolizadas pelo sistema hepático enzimático P450 poderão ser afetadas pelo alho. Além disso, os quimioterápicos poderão ter seus níveis alterados, conforme foi evidenciado no tratamento de pessoas com diagnóstico de leucemia, quando a citarabina e a fludarabina apresentaram efeito intensificado. Assim sendo, é possível que plantas medicinais ou fitoterápicos chineses possam ter efeitos semelhantes e seus efeitos farmacológicos e interações precisam ser melhor estudados. Entretanto, esta interação não foi mencionada no campo da acupuntura.

Segundo Yates et al. ${ }^{29}$ e Zhi et al. ${ }^{19}$, o uso da medicina complementar em chinesas com câncer de mama é alto, pois alcança a maioria das pessoas com câncer (91\%), concorrendo com os tratamentos convencionais. Entretanto, apesar de ser uma abordagem milenar, a acupuntura ainda é restrita a uma pequena parcela dos indivíduos. Segundo Pu et al. ${ }^{20}$, dois terços dos entrevistados em Taiwan acreditavam que a MTC poderia "curar a raiz de doenças", ao contrário do tratamento convencional. Entretanto, os médicos em Taiwan raramente conhecem a MTC, sendo incapazes de orientar tratamentos. Para Siegel et al. ${ }^{30}$, apesar da popularidade das práticas integrativas no contexto brasileiro, a maioria dos profissionais de saúde em oncologia desconhece seu potencial para a integração na atenção oncológica. Mesmo quando articulam algum apoio, este é limitado à percepção de ineficácia. Consequentemente, em sua maioria, não estão preparados para discutir ou recomendálas as pessoas com câncer.

Possivelmente a falta de diálogo com outros especialistas ou oncologistas a respeito das possibilidades de associação do tratamento não convencional limita o uso da acupuntura e perpetua o ciclo de desinformação. No Brasil, apesar dos avanços, a inserção da acupuntura na grade curricular de escolas de saúde ainda é bastante restrita, assim como o número de residências médicas ${ }^{31}$. Além 
ISSN 2179-6750

disso, o fato de ainda ser considerada uma especialidade médica contra-hegemônica ou "alternativa", cuja credibilidade quanto à cientificidade ainda é questionada, contribui para sua limitada inserção na academia e em serviços de saúde. Frequentemente os profissionais não detêm conhecimentos acerca das possibilidades da acupuntura e não oferecem atitudes de apoio para as pessoas com câncer. Este mesmo problema é enfrentado pela Homeopatia, conforme observado em estudos de representação social realizados em Vitória, ES ${ }^{32}$.

Outra questão importante são os conflitos existentes entre as diversas categorias profissionais que alegam que o uso da acupuntura deve ser multidisciplinar, enquanto que as instituições médicas reclamam a exclusividade do exercício da acupuntura por se tratar de uma especialidade e pela necessidade de elaboração de diagnóstico clínico e prescrição de tratamento ${ }^{33}$.

Segundo Spadacio e Barros ${ }^{7}$, as motivações para o uso das MAC em pessoas com câncer estão relacionadas à importância de fortalecer o sistema imunológico, aliviar os efeitos colaterais da quimioterapia, criar esperança e cura e de prevenção da recidiva da doença. Yong et al. ${ }^{18}$ concordam que a busca pela acupuntura está relacionada com a percepção de sua influência no sistema imunológico. Além disso, outros aspectos também são considerados, como o controle do estresse, o bem-estar e a melhora da qualidade de vida. A insatisfação com o tratamento oncológico também é relatada pelas pessoas com câncer. Esses fatores parecem explicar o aumento da procura pela acupuntura entre esses sujeitos, apesar do desconhecimento entre médicos.

Os estudos de prevalência realizados por Zhi et al. ${ }^{19}$ e Yong et al. ${ }^{18}$ demonstraram baixa prevalência do uso de acupuntura quando comparada com outras práticas, especialmente a fitoterapia. Tal dado pode refletir uma realidade específica, já que ambos os estudos foram realizados a partir do banco de dados do Shanghai Breast Cancer Study. Entretanto, foi considerado pelo grupo de mulheres como um tratamento eficaz, com potência para interferir no sistema imunológico e em promover a melhoria de sintomas de alta prevalência no câncer, como as algias, e os sintomas de menopausa. $\mathrm{Pu}$ et al. ${ }^{20}$, demonstraram que $22 \%$ dos participantes utilizaram a MTC e apenas $5 \%$ a acupuntura. Confirma-se o predomínio de mulheres de maior escolaridade entre os usuários da acupuntura e a propensão quanto à escolha do tratamento com acupuntura.

Esse dado corrobora com Spadacio e Barros ${ }^{7}$ que, na análise do perfil de usuários das medicinas alternativas e complementares (MAC) no Brasil, verificaram que a maioria são adultos, com idade entre 30 e 59 anos, do sexo feminino, com elevado grau de escolaridade e renda familiar alta. Além disso, Pu et al. ${ }^{20}$ referiram que a maior duração da doença implica no aumento do uso das MAC. Esse fato pode estar relacionado ao tempo necessário para obter conhecimentos e optar pelos tratamentos não convencionais, além da possibilidade de estarem relacionados à ânsia pela cura ou à 
ISSN 2179-6750

redução da confiança nos tratamentos convencionais. Spadacio e Barros ${ }^{7}$ referem que há maior percepção de risco de morte ou retorno da doença entre os usuários com câncer que usam as MAC, ou seja, quanto maior o estresse mental, maior a prevalência do uso. Entretanto, percebem o seu uso de forma benéfica, desprovidas de toxicidade e com potencial para proporcionarem mudanças no estilo e na qualidade de vida, influenciando positivamente nos rumos da doença.

A inclusão do artigo descritivo com metodologia qualitativa de Peter et al. ${ }^{23}$ pode ampliar o universo de percepções acerca do tratamento com acupuntura em mulheres com câncer de mama. Esse estudo faz referência aos seus benefícios em diversos sintomas que refletem na melhoria da qualidade de vida, como humor e disposição geral. Destaca-se que os dados apresentados demonstram um grande potencial relativo ao tratamento com acupuntura para mulheres desesperançadas e portadoras de fadiga, sintoma de difícil controle pelo tratamento convencional. Esses relatos também contribuem para melhor compreender a subjetividade das participantes, aspectos pouco explorados em estudos com outros tipos de metodologia. Assinala também a frustração das mulheres do grupo controle que já passaram pela quimioterapia e que buscam alternativas para o controle dos sintomas residuais, prevenção de recidivas e melhor qualidade de vida. Desenhos que permitam o tratamento com acupuntura no grupo controle após a análise dos dados devem ser incentivados, considerando a importância de promover atenção e cuidado humanizado e ético em pesquisas.

\section{Conclusão}

Atualmente os estudos sobre o uso da acupuntura no câncer de mama são realizados com objetivo de controlar os sintomas da doença e de minimizar o impacto dos efeitos adversos do tratamento oncológico, apresentando resultados bastante promissores. Os artigos incluídos nesta revisão demonstraram a efetividade da acupuntura no tratamento do estresse e da ansiedade de mulheres com câncer de mama, tanto no período peroperatório como também durante a quimioterapia. Ou seja, apesar da limitação da amostragem e do número de estudos, evidenciou-se que a acupuntura é uma abordagem holística que também pode ser útil em hospitais, tanto no peroperatório como nos centros oncológicos, uma vez que, além das melhoras observadas, traz bemestar e provoca menos efeitos secundários do que os tratamentos convencionais.

Os estudos de prevalência demonstraram que a maioria das chinesas com câncer de mama utiliza a MTC associada a outros tratamentos ocidentais, embora a acupuntura seja pouco usada. A desinformação entre os oncologistas ainda perdura e precisa ser mais bem compreendida.

Tendo em vista a importância do câncer de mama para a saúde pública e a alta prevalência em todo o mundo, ressalta-se a urgência de novas investigações. A realização de pesquisas que avaliem 
ISSN 2179-6750

a efetividade da acupuntura ao longo de todas as etapas do tratamento oncológico da mulher com câncer de mama, com desenhos mais robustos, e estudos de coorte que possam avaliar as taxas de recidiva e a sobrevida de usuários da acupuntura associada ao tratamento oncológico é indicada. Além disso, é importante promover medidas de aproximação entre os mastologistas, oncologistas e acupunturistas a fim de compartilharem experiências e saberes, considerando a perspectiva de melhorar a atenção e o cuidado às pessoas com câncer. Por fim, ressalta-se a importância de novos estudos que subsidiem informações relativas ao custo e efetividade, a fim de contribuir para a implementação de políticas públicas no intuito de ampliar o acesso a esse tratamento à população.

\section{Referências}

1. Instituto Nacional de Câncer (INCA). Controle do câncer de mama: prevenção [Internet]. Rio de Janeiro: INCA; 1996 [atualizado 1 dez 2015; acesso em 10 out 2016]. Disponível em:

http://www2.inca.gov.br/wps/wcm/connect/acoes_programas/site/home/nobrasil/programa_ controle_cancer_mama/prevencao

2. World Health Organization. Traditional Medicine Strategy: 2014-2023. Geneva: WHO; 2013.

3. Frass M, Strassl RP, Friehs H, Müllner M, Kundi M, Kaye AD. Use and acceptance of complementary and alternative medicine among the general population and medical personnel: a systematic review. Ochsner J. 2012 Spring;12(1):45-56.

4. Lin JG, Chen YH. The role of acupuncture in cancer supportive care. Am J Chin Med. 2012;40(2):219-29. https://doi.org/10.1142/S0192415X12500176.

5. Ezzo JM, Richardson MA, Vickers A, Allen C, Dibble SL, Issell BF, et al. Acupuncturepoint stimulation for chemotherapy-induced nausea or vomiting. Cochrane Database Syst Rev. 2006 Apr 19;(2):CD002285. https://doi.org/10.1002/14651858.CD002285.pub2.

6. NIH Consensus Conference. Acupuncture. JAMA. 1998 Nov 4:280(17):1518-24. https://doi.org/10.1001/jama.280.17.1518.

7. Spadacio C, Barros NF. Uso de medicinas alternativas e complementares por pacientes com cancer: revisão sistemática. Rev Saude Public. 2008 fev;42(1):158-64. http://dx.doi.org/10.1590/S0034-89102008000100023.

8. O'Regan D, Filshie J. Acupuncture e Câncer. Auton Neurosci. 2010 Oct 28:157(1-2):96100. https://doi.org/10.1016/j.autneu.2010.05.001.

9. Rocha DK, Tolentino BG, Genschow FCZ, Sampaio FC. Acupuntura médica no Brasil: um 159 
ISSN 2179-6750

breve histórico. [Brasília, DF]: CMBA; 2008.

10. Brasil. Ministério da Saúde. Política Nacional de Práticas Integrativas e Complementares (PNPIC). Brasília, DF; 2006 [acesso em 19 jun 2016]. Disponível em:

http://dab.saude.gov.br/portaldab/pnpic.php

11. Brasil. Ministério da Saúde. Núcleo de Práticas Integrativas e Complementares do Departamento de Atenção Básica. Boletim de monitoramento das ações de Práticas Integrativas e Complementares (PIC). Brasília, DF; 2016 [acesso em 20 jun 2006]. Disponível em: http://blog.atencaobasica.org.br/wp-content/uploads/2016/06/InformePICS.pdf

12. Santos MC, Tesser CD. Um método para a implantação e promoção de acesso às Práticas Integrativas e Complementares na Atenção Primária à Saúde. Cienc Saude Colet. 2012 nov; 17(11):3011-24. http://dx.doi.org/10.1590/S1413-81232012001100018.

13. Santos BS. Um discurso sobre as ciências. Porto: Editora Afrontamento; 1987. 59 p.

14. Cormanique TF, Almeida LED, Rech CA, Rech D, Herrera ACSA, Panis C. Estresse psicológico crônico e seu impacto no desenvolvimento de neoplasia mamária agressiva. Einstein (São Paulo). 2015 jul-set;13(3):352-6. http://dx.doi.org/10.1590/S1679$45082015 \mathrm{AO} 3344$.

15. Baqutayan S. The effect of anxiety on breast cancer patients. Indian J Psychol Med. 2012 Apr-Jun; 34(2):119-123. http://dx.doi.org/10.4103/0253-7176.101774.

16. Primo CC, Gonçalves LRN, Olympio PCAP, Leite FMC, Amorim MHC. Ansiedade em mulheres com câncer de mama. Enferm Glob. 2012 out;(28):63-73.

17. Mendes KDS, Silveira RCCP, Galvão CM. Revisão integrativa: método de pesquisa para a incorporação de evidência na saúde e na enfermagem. Texto Context Enferm. 2008 Out-dez;17(4):758-64. http://dx.doi.org/10.1590/S0104-07072008000400018.

18. Yong C, Xiao-Ou S, Yutang G, Wanqing W, Zhi-Xian R, Fan J, et al. Use of complementary and alternative medicine by Chinese women with breast cancer. Breast Cancer Res Treat. 2004;85(3):263-270. http://dx.doi.org/10.1023/B:BREA.0000025422.26148.8d.

19. Zhi C, Kai G, Ying Z, Wei Z, Wei L, Xiao-Ou S. The Use of complementary and alternative medicine among Chinese women with breast cancer. J Altern Complement Med. 2008 Oct;14(8):1049-55. http://dx.doi.org/10.1089/acm.2008.0039.

20. Pu C-Y, Lan VM, Lan CF, Lang H-C. The determinants of traditional Chinese medicine and acupuncture utilization for cancer patients with simultaneous conventional 
ISSN 2179-6750

treatment. Eur J Cancer Care (Engl). 2008 Jul;17(4):340-9. doi:

https://doi.org/10.1111/j.1365-2354.2007.00865.x.

21. Mallory MJ, Croghan KA, Sandhu NP, Lemaine V, Degnim AC, Bauer BA, et al. Acupuncture in the postoperative setting for breast cancer patients: a feasibility study. Am J Chin Med. 2015;43(1):45-56. https://doi.org/10.1142/S0192415X15500032.

22. Tas D, Uncu D, Sendur MA, Koca N, Zengin N. Acupuncture as a complementary treatment for cancer patients receiving chemotherapy. Asian Pac J Cancer Prev. 2014 Jul;15(7):3139-44. http://dx.doi.org/10.7314/APJCP.2014.15.7.3139.

23. Peter M, Joy B, Jacqueline F, Jennifer FJ, Alexander M. Receiving or not receiving acupuncture in a trial: the experience of participants recovering from breast cancer treatment. Complement Ther Clin Pract. 2014 Nov;20(4):291-6. http://dx.doi.org/10.1016/j.ctcp.2014.10.002. Epub 2014 Oct 18.

24. MacPherson H, Altman DG, Hammerschlag R, Youping L, Taixiang AW, Moher D. Revised Standards of Reporting Interventions in Clinical Trials of Acupuncture (STRICTA): extending the CONSORT statement. PLoS Med. 2010 Jun 8;7(6):e1000261. http://dx.doi.org/10.1371/journal.pmed.1000261.

25. Acar HV, Cuvaş O, Ceyhan A, Dikmen B. Acupuncture on Yintang point decreases preoperative anxiety. J Altern Complement Med. 2013 May;19(5):420-4. http://dx.doi.org/10.1089/acm.2012.0494. Epub 2012 Dec 27.

26. Wang SM, Peloquin C, Kain ZN. The use of auricular acupuncture to reduce preoperative anxiety. Anesth Analg. 2001 Nov;93(5):1178-80. http://dx.doi.org/10.1097/00000539-200111000-00024.

27. Pilkington K, Kirkwood G, Rampes H, Cummings M, Richardson J. Acupuncture for anxiety and anxiety disorders - a systematic literature review. Acupunct Med. 2007;25(1-2):1-10. http://dx.doi.org/10.1136/aim.25.1-2.1.

28. Ernst E, Cassileth BR. The prevalence of complementary/alternative medicine in cancer: a systematic review. Cancer. 1998 Aug 15;83(4):777-82.

29. Nicoletti MA, Oliveira-Júnior, MA, Bertasso, CC, Caporossi PY, Tavares, APL. Principais interações no uso de medicamentos fitoterápicos. Infarma. 2007 [acesso 22 set. 2016];19(1-2):32-40. Disponível em: http://www.cff.org.br/sistemas/geral/revista/pdf/10/infa09.pdf. Acesso em 22 set. 2016.

30. Yates JS, Mustian KM, Morrow GR, Gillies LJ, Padmanaban D, Atkins JN, et al. Prevalence of complementary and alternative medicine use in cancer patients during 161 
ISSN 2179-6750

treatment. Support Care Cancer. 2005 Oct;13(10):806-11. http://dx.doi.org/10.1007/s00520004-0770-7.

31. Siegel P, Broom A, Bowden V, Adams J, Barros NF. Attitudes toward complementary and alternative medicine amongst oncology professionals in Brazil. Complement Ther Med. 2016;27:30-4. https://doi.org/10.1016/j.ctim.2016.04.003

32. Costi JM1, Silva JB, Min LS, Moré AO, Hokama AL. Acupunct Med. 2012 Dec;30(4):3503. Http://dx.doi.org/10.1136/acupmed-2012-010184.

33. Figueiredo TAM, Machado VLT. Representações sociais da homeopatia: uma revisão de estudos produzidos no Estado do Espírito Santo. Cienc Saude Colet. 2011;16(Suppl 1):9991005. http://dx.doi.org/10.1590/S1413-81232011000700032. 